\title{
- Cancer Stem Cell Column. \\ Technology update for the sorting and identification of breast cancer stem cells
}

\author{
Jian Chen ${ }^{1,2}$, Zeng-Liang Chen ${ }^{1}$ \\ ${ }^{1}$ School of Medicine, Zhejiang University, Hangzhou, Zhejiang 310058, P. R. China; ${ }^{2}$ Zhejiang Medical College, Hangzhou, Zhejiang 310053 , P. R. \\ China
}

[Abstract) Breast cancer stem cells are a group of undifferentiated cells with self -renewal and multidifferentiation potential. Chemotherapeutic and radiotherapeutic resistance, hypoxic resistance, high tumorigenicity, high cell invasion, and metastatic abilities are characteristics of these cells, which are responsible for breast cancer recurrence. Therefore, the correct sorting and identification of breast cancer stem cells is a primary step for research in this field. This article briefly describes the recent progress on sorting and identification technologies for breast cancer stem cells. Sorting technologies include the side population technique, technologies that depend on cell surface markers, ALDEFLUOR assays, and in situ detection. Identification technologies include mammosphere cultures, limited dilution in vitro, and in-vivo animal models. This review provides an important reference for breast cancer stem cell research, which will explore new methods for the treatment of patients with breast cancer.

Key words: Breast neoplasm, stem cell, cell surface maker, sorting

Stem cells are a class of cells with the capacities of self-renewal and the potential for multidifferentiation. In normal situations, the abilities of self-renewal and differentiation are strictly regulated. Once the regulatory mechanism is disrupted, cells will grow and proliferate, finally forming tumors. Hamburger et al. ${ }^{[1]}$ proposed the concept of cancer stem cells based on stem cell theory. Cancer stem cells are considered to be a group of special cells that are present in tumor cells with the characteristics of self-renewal, multidifferentiated potential, and high tumorigenicity. Cancer stem cells resist cell apoptosis induced by chemotherapy and radiotherapy, and have high metastastic ability. Conventional treatment cannot eradicate cancer stem cells, and therefore lead to the recurrence of cancer. Breast cancer is a common malignant cancer in women. Worldwide epidemiologic studies show that the morbidity and mortality rates of breast cancer are the highest among all malignant cancers in women. In our country, the incidence of breast cancer has increased gradually, and has become one of the malignant cancers that seriously affects the physical and mental health of women, even their lives. In 2003, Al-Hajj et al. ${ }^{[2]}$ isolated breast stem cells from breast cancer tissue for the first

Correspondence to: Zeng-Liang Chen; Tel: +86-29-87679366;

Email:wopu1998@hotmail.com

This paper was translated from Chinese into English by CJC Medical Translation and edited by Hope J. Lafferty on 2010-01-17.

The Chinese version of this paper is avaiable at http://www.cjcsysu.cn/cn/article .asp?id=16694.

Received: 2009-11-04; Accepted: 2009-12-28 time, which provided novel thinking for breast cancer therapy. Therefore, how to isolate breast cancer stem cells and the study of the possible regulatory mechanisms of breast cancer stem cells have important significance in identifying the pathogenesis and radical cure of breast cancer. In this article, we reviewed the commonly used sorting and identification techniques for breast cancer stem cells.

\section{Sorting breast cancer stem cells}

\section{The side population sorting technique}

Hoechst 33342 is a kind of DNA supplemental dye, which binds with AT-rich canaliculus of DNA. Studies have shown that using Hoechst 33342 to stain stem cells, a group of cells are stained dark by flow cytometry assays, called a side population $(\mathrm{SP})^{[3]}$. This separation technique is called side population sorting, and the principle is that the transmembrane channels on the stem cell membrane, such as ATP-binding cassette super-family G member 2 (ABCG2) or breast cancer resistance protein 1 (BCRP1), can pump the Hoechst 33342 and rhodamine 123 out of cells and the cells appear weakly stained. Through 355-nm ultraviolet (UV) excitation by flow cytometry, cells are detected or isolated.

Patrawala et al. ${ }^{[4]}$ isolated cancer stem cells from human tumor cells by side population analysis in the breast cancer cell line MCF-7. The percent of the side population was $0.2 \%$. Compared with non-side population cells, the tumorigenicity was higher in the side population cells, with fewer cells required for 
tumor formation and a shorter latent period. Many examples have been shown by side population analysis for successful isolation. However, a few flaws still exist, which limit the application of the technique. First, because Hoechst is toxic to cells and results in some false phenomena, such as lower self-renewal and tumorigenicity of the main population cells, positive cells do not proliferate in vitro or do not form tumors in vivo after sorting. Second, studies have proved that sorted side population cells do not have stem cell activities ${ }^{[5]}$.

\section{Expression of cell surface markers}

Cell surface markers have been widely used for sorting cancer stem cells. Different tissue and species tumors have different cancer stem cell markers. Below, we introduce some recently studied surface markers of breast cancer stem cells.

$C D 44^{+} / C D 244^{-H o w} /$ lin - In 2003, Al-Hajj et al. ${ }^{[2]}$ found that CD44 ${ }^{+}$CD24/llow/Lin- was a good marker for distinguishing the tumorigenicity of cells. They randomly selected 9 patients with breast cancer, and isolated cells with $\mathrm{CD}_{4} 4^{+} / \mathrm{CD} 24^{- \text {-low }} / \mathrm{Lin}^{-}$in 8

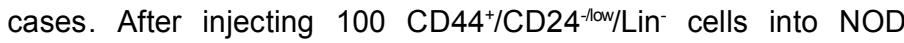
SCID mice, all mice formed tumors. Injecting $2 \times 10^{4}$ cells without this phenotype into NOD SCID mice failed to form tumors. In addition, they isolated $\mathrm{CD}^{2} 4^{+} / \mathrm{CD} 24^{-1 / \mathrm{low} / \mathrm{Lin}^{-}}$cells from the xenografts formed by injecting CD44 $/$ CD24-low/Lin- cells into NOD SCID mice and reinjected 100 of these cells into NOD SCID mice, where tumors also formed. According to the cycle of isolation-injection-tumorigenesis, after four generations, the tumorigenicity did not reduce, and cells showed the characteristics of self-renewal and differentiation, which were considered breast cancer stem cells.

CD44 ${ }^{+} / \mathrm{CD} 24^{-1 / \mathrm{low}} / \mathrm{Lin}^{-}$has also been used in isolating other types of cancer stem cells ${ }^{[6,7]}$. However, this method has deficiencies, such as poor homogeneity of the isolated cancer stem cells, which can be further divided into different subgroups. Some of these subgroups did not form tumors, suggesting that these cells are not complete breast cancer stem cells by using the molecular markers selected ${ }^{[8]}$. Furthermore, the molecular markers exhibit some differences in different tumor types. For example, in pancreatic cancer, the marker of stem cells with self-renewal and multidifferentiated potential is $\mathrm{CD} 44^{+} / \mathrm{CD} 24^{+[9]}$.

ITGA6/ 6 6-integrin Purushotham et al. ${ }^{[10]}$ have found that a subpopulation of MCF-7 cells overexpressed a 6-integrin. This subpopulation was capable of growth in anchorage-independent conditions as spherical organoids and displayed resistance to proapoptotic agents and significantly greater tumorigenicity than its parental line, with as few as 1000 cells able to form tumors in SCID mice. Silencing ITGA gene by RNA interference (RNAi) caused mammosphere-derived cells to lose their ability to grow as mammospheres and abrogated their tumorigenicity in mice. They showed that $\alpha 6$-integrin was a potential therapeutic target aimed at tumor-generating subsets of breast cancer cells.

CD133/PROM1/prominin CD133 glucoprotein is a cell surface protein with a molecular weight of $120 \mathrm{kDa}$ and five transmembrane domains. CD133 expression decreases rapidly with cell differentiation ${ }^{[11]}$. Thus, it can be used as a molecular marker for isolating and identifying stem and progenitor cells.
Singh et al. ${ }^{[12]}$ used CD133 as a molecular marker to isolate cancer stem cells from brain cancer for the first time.

Wright et al. ${ }^{[13]}$ injected BRCA1-knockout breast cancer cells into mouse breast fat pads, after tumorigenesis, isolated a series of tumor cells from xenografts, and found that CD $133^{+}$cells had the characteristics of cancer stem cells like CD44 $/$ CD24-low cells. Thus, they proposed that cancer stem cells also were not homogeneous. This was the first report where CD133 was positive in breast cancer stem cells. Though cancer stem cells are successfully isolated from brain, prostate, and colon cancers by using CD133 as a marker ${ }^{[12,14,15]}$, no reports have shown that CD133 is a marker for the isolation of breast cancer stem cells.

CD29/31-integrin and CD61/33-integrin CD29 and CD61 are two surface proteins expressed in breast luminal cells. In breast undifferentiated CD24 ${ }^{+}$cells, CD29 ${ }^{\text {low }}$ cells are most common, and CD29 high cells are breast cancer stem cells ${ }^{[16]}$. According to CD61 expression, $C D 24^{+} \mathrm{CD} 29^{\text {low }}$ cells can be divided into two types: CD61 ${ }^{+}$luminal progenitor cells and CD61- mature luminal cells ${ }^{[17]}$. In MMTV-WNT1 tumor mouse models, the tumorigenicity of $\mathrm{CD} 1^{+}$cells isolated through flow cytometry is greater than CD61cells ${ }^{[18]}$.

Many types of cancer stem cells are isolated from mouse models and in humans through applying cell surface specific molecules and flow cytometry. Many cell surface markers in the mouse breast are used for isolating breast cancer stem cells, and the isolated cells are confirmed by several methods as stem cells. However, few molecular markers are used for specifically isolating breast cancer stem cells from human tissue, and the analytic standard is difficult to identify. Successful mouse models are difficult to apply to humans. Therefore, it is essential to review the widely used markers for sorting primary breast cancer stem cells in humans.

\section{ALDEFLUOR assay}

ALDEFLUOR assay is based on detecting ALDH1 activity. ALDH1 oxidizes retinol to retinoic acid. Cells with highly activated ALDH1 are labeled with fluorescence and can be detected and selected. ALDH is overexpressed in all living primary hematopoietic cells. The substrate of ALDH, BAAA (Bodipy-aminoacetaldehyde), can be transformed to the fluorescent product BAA (Bodipy-aminoacetate). Cells with high ALDH expression appear bright fluorescence (ALDHbr), which can be detected, evaluated, and isolated by flow cytometry. $\mathrm{ALDH}^{\text {br }}$ cells include $\mathrm{CD}^{+} 4^{+}$cells, $\mathrm{CD} 133^{+}$cells, $\mathrm{Kit}^{+}$cells, $\mathrm{Lin}^{-}$ cells, colony-forming cells and so on.

In mouse and human hematopoietic cells, neural stem cells, and progenitor cells, ALDH1 is high activated ${ }^{[19]}$. Transplanting 10 ALDH-positive cells isolated from a rat hematopoietic system to a mouse that is irradiated by half-lethal doses of radio rays, cells have the capacity for long-term existence in mouse bone marrow. Studies have shown that ALDEFLUOR-positive cells isolated from the mouse brain have self-renewal abilities and form neural stem cells and neural epithelium-like stem cells ${ }^{[20]}$. After in vitro culture, these cells differentiate to neurons and glia. Compared with ALDH-negative cells, the survival rate of positive cells is higher after transplantion to the brain. 
Using the same method, stem and progenitor cells have been successfully isolated from breast tissue. Ginestier et al. ${ }^{[8]}$ detected whether these tumor cells form breast tumors in NOD SCID mice. The results showed that only ALDH1-positive cells form tumors; as few as 500 cells led to tumorigenesis. Conversely, 50000 ALDH-negative cells did not cause tumor formation.

However, the ALDEFLUOR assay has some limitations. For example, in H522 cells, ALDHbr and ALDHlow cells form tumors in NOD SCID mice, and tumors caused by ALDH ${ }^{\text {low }}$ cells grow bigger and more quickly ${ }^{[21]}$. These results have indicated that in lung cancer, ALDEFLUOR-positive cells were not completely stem cells. Cells were heterogeneous, and other markers are needed to distinguish further. In breast cancer cells, ALDEFLUOR-positive cells can be further detected by molecular markers such as $\mathrm{CD}_{4} 4^{+}, \mathrm{CD} 24$, and $\mathrm{CD} 133$. Among them, the tumorigenicity of the ALDEFLUOR $/ C D 44^{+} / C D 24^{-}$and ALDEFLUOR ${ }^{+} / \mathrm{CD}_{4} 4^{+} / \mathrm{CD} 133^{+}$subpopulations are the highest ${ }^{[22]}$.

\section{In situ detection}

Acetaldehyde dehydrogenase activity is mainly due to its major form ALDH1A1. ALDH1A1 is highly expressed in stem cells and plays regulatory roles in stem cells ${ }^{[23]}$. Breast cancer tissue samples are fixed by methanal, embedded in paraffin, and stained by ALDH1A1 in situ, where normal stem cells and breast cancer stem cells can be identified. Thirty percent of breast cancers have a small amount of ALDH1A1-positive cells, and through analyzing the expression of molecular markers of stem and progenitor cells in human breast cancer tissue, prognosis can be predicted ${ }^{[8]}$.

Using immunohistochemistry to examine ALDH1A expression of stem cells in situ is a simple and effective method. However, this method is still disputed. In transgenic mice, knockout ALDH1A does not affect the function of the hemopoietic system or hemopoietic stem cells. This is probably due to other forms of ALDH that are present, for example ALDH1A3, which plays an important role in maintaining hemopoietic cell function ${ }^{[24]}$.

Another in situ detection is CD44 and CD24 double staining. CD $44^{+} / C D 24^{\text {low }}$ cells are stem cells. In paraffin-embedded tumor tissue, the rate of $\mathrm{CD} 44^{+} / \mathrm{CD} 24^{\text {low }}$ cells is lower than $10 \%$, and the results are not related to clinicopathologic characteristics or clinical results ${ }^{[25]}$. Studies have shown that $\mathrm{CD} 44^{+} / \mathrm{CD} 24^{\text {low }}$ cells commonly appear in basal-like subtype tumors, and secondly in BRCA1 hereditary tumors. While in ERBB2 positive tumors, nearly no $\mathrm{CD} 44^{+} / \mathrm{CD} 24^{\text {low }}$ cells were found ${ }^{[26]}$.

Although the $\mathrm{CD} 44^{+} / \mathrm{CD} 24^{\text {low }}$ phenotype is the most important marker in breast cancer stem cells, CD $44^{+} / \mathrm{CD} 24^{\text {low }}$ still not used for clinical research. Dontu et al. ${ }^{[2]}$ have pointed out that the application of these markers faces a lot of questions: whether $\mathrm{CD} 44^{+} / \mathrm{CD} 24^{\text {low }}$ is related to stem cells only in specific breast cancers, whether other cancer stem cells exist in tumors without $\mathrm{CD} 44^{+} / \mathrm{CD} 24^{\text {low }}$ cells, whether the origin is the same, and whether CD44+/CD24low dynamically changes tumor development. Therefore, for this method to be used in clinical applications, the molecular marker for isolating cancer stem cells requires a verification process.

\section{Identification of breast cancer stem cells}

Cells separated and isolated by various methods need to be identified whether they are cancer stem cells. Similar to the functional definition of cancer stem cells, recently the identification of breast cancer stem cells is determined by self-renewal, differentiation abilities, and tumorigenicity. The methods involve in-vitro cell cultures and in-vivo animal models.

\section{In vitro cell culture identification}

Mammospheres culture Dontu et al. ${ }^{[28]}$ have proved that in normal breast tissue, human breast stem and progenitor cells can have growth suspended, and form mammospheres involving stem and progenitor cells. These nonadherent mammospheres involve amounts of early-stage stem and progenitor cells that have the specialized functions of stem cells, such as self-renewal, multidifferentiation potential, and breast tissue regeneration.

As stem and progenitor cells can be cultured in non-serum media, long-cultured stem and progenitor cells were selected from breast tissue. Breast cancer cells were cultured in non-serum media with fibroblast growth factor and epidermal growth factor, and mammospheres (tumor spheres) formed. These mammospheres contained CD $44^{+} / \mathrm{CD} 24^{\text {low }}$ cells and did not express differentiated markers, such as markers of the luminal epithelium and myoepithelium. They also expressed angiogenesis, cytoprotection-related factors, and stem cell marker OCT4. Under differentiated conditions, cells received the multidifferentiated potential and formed tumors in NOD SCID mice $^{[29]}$.

The hedgehog signaling, BMI/polycomb, and Notch signaling pathways play important roles in self-renewal and differentiation of stem and progenitor cells in human breast development ${ }^{[30]}$. The malignant mutations of early-stage breast cancer are related to the disorders of the Notch signaling pathway. Blocking the Notch signaling pathway by DAPT or Notch4 counteractive antibodies, the number of breast mammospheres originating from pre-invasive forms of primary breast cancer cells obviously decreases ${ }^{[31]}$.

Enrichment of breast cancer stem cells by mammosphere culture is a common method for in-vitro breast cancer stem cell culturing. This method is not only an important and novel tool for studying the characteristics of breast cancer stem cells, but also has important significance in studying the key signaling pathways of normal and cancer stem cells.

Limited dilution assay Limited dilution assay is an effective method for detecting the proliferation capacity of single breast cancer cells. Like the heterogeneity of tumor cells, the potential for colony formation of breast cancer cell groups are different, and the potential for proliferation is also different. Generally, breast cancer cells with the ability to form colonies are called breast cancer stem cells. Soft agar assays are often used in colony formation assays. A single breast cancer cell is cultured in plates treated with soft agar, and cells with a continuous capacity for colony formation are selected. Cells are then further amplified 


\section{Chinese Journal of Cancer}

for in-vivo and in-vitro experiments. Finally, the cell surface markers are identified. Usually in-vitro colony formation is consistent with tumorigenicity induced by transplantation.

\section{In-vivo animal model identification}

Various selected breast cancer subpopulations have been injected into animals according to different cell concentrations. Then the tumorigenicity of these cells and select surface markers of predominant cells were observed. Also by combining several predominant cell surface markers to select various combinations of breast cancer cell groups, these cells are injected into animals according to different cell concentrations to observe the tumorigenicity to determine the target cells. Breast cancer stem cells with $\mathrm{ESA}^{+} \mathrm{CD} 44^{+} / \mathrm{CD} 24^{-1 / \mathrm{low}} / \mathrm{Lin}^{-}$are the only cells that can form tumors in continuous transplantation. As few as 100 to 200 cells can form tumors in the mouse breast ${ }^{[2]}$.

\section{Prospects}

The stem cell theory of cancer and the discovery of cancer stem cells provide novel ways to think about cancer therapy. Cancer stem cells have been isolated from many cancer tissues and cell lines by several methods. Cancer stem cell theory holds important significance in studying the biologic action, regulatory mechanisms, and targeting therapy of cancer stem cells.

Breast cancer stem cells are considered to be the origin of resistance to breast cancer treatment and recurrence. Therefore, the study of breast cancer stem cells is important in finding a radical cure for breast cancer. Sorting and identifying breast cancer stem cells are the primary steps in the study of the whole breast cancer stem cell system, which relates to the further study of breast cancer stem cell microenvironments, abnormal signal transduction, and exploring regulatory molecules of cancer stem cells. In past studies, the technologies for the sorting and identification of breast cancer stem cells have progressed quickly. Specifically, the application and renewal of cell surface markers are essential for the in-depth study of breast cancer stem cells. However, there are still questions. The number of breast cancer stem cells is small in breast tissue. Differences exist in recognizing the specific cell surface markers. The technique for isolating breast cancer stem cells is immature. Isolating single breast cancer stem cells often disturbs normal breast stem cells. These are just a few examples.

The study of the microenvironment of breast cancer stem cells is not in-depth enough. The molecular mechanisms of self-renewal, asymmetric division, and microenvironment maintenance are the main focus of the recent research on breast cancer stem cells. Many related studies have just started, so the theories cannot used for clinical treatment yet and further studies are very important.

Along with the deep understanding of the biology and regulatory mechanisms of breast cancer stem cells, cancer treatment modalities may change from killing tumor cells to treating cancer stem cells. The discovery of drugs that kill cancer stem cells directly will apply a qualitative evolution in cancer therapy and open a new chapter.

\section{References}

[1] Hamburger AW, Salmon SE. Primary bioassay of human tumor stem cells [J]. Science, 1977, 197(4302): 461-463.

[2] Al-Hajj M, Wicha MS, Benito-Hernandez A, et al. Prospective identification of tumorigenic breast cancer cells [J]. Proc Natl Acad Sci U S A, 2003, 100(7): 3983-3988.

[3] Goodell MA, Brose K, Paradis G, et al. Isolation and functional properties of murine hematopoietic stem cells that are replicating in vivo [J]. J Exp Med, 1996, 183(4):1797-1806.

[4] Patrawala L, Calhoun T, Schneider-Broussard R, et al. Side population is enriched in tumorigenic, stem-like cancer cells, whereas ABCG2 + and ABCG2- cancer cells are similarly tumorigenic [J]. Cancer Res, 2005, 65 (14): 6207-6219

[5] Montanaro F, Liadaki K, Schienda J, et al. Demystifying SP cell purification: viability, yield, and phenotype are defined by isolation parameters [J]. Exp Cell Res, 2004, 298(1): 144-154.

[6] Dalerba P, Dylla SJ, Park IK, et al. Phenotypic characterization of human colorectal cancer stem cells [J]. Proc Natl Acad Sci U S A, 2007, 104 (24): 10158-10163.

[7] Prince ME, Sivanandan R, Kaczorowski A, et al. Identification of a subpopulation of cells with cancer stem cell properties in head and neck squamous cell carcinoma [J]. Proc Natl Acad Sci U S A, 2007, 104(3): 973-978.

[8] Ginestier C, Hur MH, Charafe-Jauffret E, et al. ALDH1 is a marker of normal and malignant human mammary stem cells and a predictor of poor clinical outcome [J]. Cell Stem Cell, 2007, 1(5): 555-567.

[9] Li C, Heidt DG, Dalerba P, et al. Identification of pancreatic cancer stem cells [J]. Cancer Res, 2007, 67(3): 1030-1037.

[10] Cariati M, Naderi A, Brown JP, et al. Alpha-6 integrin is necessary for the tumourigenicity of a stem cell-like subpopulation within the MCF7 breast cancer cell line [J]. Int J Cancer, 2008, 122(2): 298-304.

[11] Peichev M, Naiyer AJ, Pereira D, et al. Expression of VEGFR-2 and AC133 by circulating human CD34 $(+)$ cells identifies a population of functional endothelial precursors [J]. Blood, 2000, 95(3): 952-958.

[12] Singh SK, Clarke ID, Terasaki M, et al. Identification of a cancer stem cell in human brain tumors [J]. Cancer Res, 2003, 63(18): 5821-5828.

[13] Wright $\mathrm{MH}$, Calcagno AM, Salcido CD, et al. Brca1 breast tumors contain distinct $\mathrm{CD} 44^{+} / \mathrm{CD} 24^{-}$and $\mathrm{CD} 133^{+}$cells with cancer stem cell characteristics [J]. Breast Cancer Res, 2008, 10(1): R10.

[14] Collins AT, Berry PA, Hyde C, et al. Prospective identification of tumorigenic prostate cancer stem cells [J]. Cancer Res, 2005, 65(23): $10946-10951$.

[15] O’Brien CA, Pollett A, Gallinger S, et al. A human colon cancer cell capable of initiating tumour growth in immunodeficient mice [J]. Nature, 2007, 445(7123): 106-110.

[16] Shackleton M, Vaillant F, Simpson KJ, et al. Generation of a functional mammary gland from a single stem cell [J]. Nature, 2006, 439(7072): 8488.

[17] Asselin-Labat ML, Sutherland KD, Barker H, et al. Gata-3 is an essential regulator of mammary-gland morphogenesis and luminal-cell differentiation [J]. Nat Cell Biol, 2007,9(2):201-209.

[18] Vaillant F, Asselin-Labat ML, Shackleton M, et al. The mammary progenitor marker CD61/beta3 integrin identifies cancer stem cells in mouse models of mammary tumorigenesis [J]. Cancer Res, 2008, 68(19): $7711-7717$

[19] Armstrong L, Stojkovic M, Dimmick I, et al. Phenotypic characterization of murine primitive hematopoietic progenitor cells isolated on basis of aldehyde dehydrogenase activity [J]. Stem Cells, 2004, 22 (7): $1142-$ 1151.

[20] Corti S, Locatelli F, Papadimitriou D, et al. Identification of a primitive brain-derived neural stem cell population based on aldehyde dehydrogenase activity [J]. Stem Cells, 2006, 24(4): 975-985.

[21] Ucar D, Cogle CR, Zucali JR, et al. Aldehyde dehydrogenase activity as a functional marker for lung cancer [J]. Chem Biol Interact, 2009, 178(13): $48-55$ 


\section{Chinese Journal of Cancer}

[22] Croker AK, Goodale D, Chu J, et al. High aldehyde dehydrogenase and expression of cancer stem cell markers selects for breast cancer cells with enhanced malignant and metastatic ability [J]. J Cell Mol Med, 2008.

[23] Duester G. Families of retinoid dehydrogenases regulating vitamin A function: production of visual pigment and retinoic acid [J]. Eur $\mathrm{J}$ Biochem, 2000, 267(14):4315-4324.

[24] Levi BP, Yilmaz $\mathrm{OH}$, Duester $\mathrm{G}$, et al. Aldehyde dehydrogenase $1 \mathrm{a} 1$ is dispensable for stem cell function in the mouse hematopoietic and nervous systems [J]. Blood, 2009, 113(8): 1670-1680.

[25] Abraham BK, Fritz P, McClellan M, et al. Prevalence of CD44+/CD24-How cells in breast cancer may not be associated with clinical outcome but may favor distant metastasis [J]. Clin Cancer Res, 2005, 11 (3): $1154-$ 1159.

[26 ] Honeth G, Bendahl PO, Ringner M, et al. The CD44 + CD24- phenotype is enriched in basal-like breast tumors [J]. Breast Cancer Res, 2008, 10(3): R53.
[27] Dontu G. Breast cancer stem cell markers-the rocky road to clinical applications [J]. Breast Cancer Res, 2008, 10(5): 110.

[28] Dontu G, Abdallah WM, Foley JM, et al. In vitro propagation and transcriptional profiling of human mammary stem/progenitor cells [J]. Genes Dev, 2003, 17(10): 1253-1270.

[29] Ponti D, Costa A, Zaffaroni N, et al. Isolation and in vitro propagation of tumorigenic breast cancer cells with stem/progenitor cell properties [J]. Cancer Res, 2005, 65(13): 5506-5511.

[30] Dontu G, Jackson KW, McNicholas E, et al. Role of Notch signaling in cell-fate determination of human mammary stem/progenitor cells [J]. Breast Cancer Res, 2004, 6(6): R605-615.

[31] Farnie G, Clarke RB, Spence K, et al. Novel cell culture technique for primary ductal carcinoma in situ: role of Notch and epidermal growth factor receptor signaling pathways [J]. J Natl Cancer Inst, 2007, 99(8): 616-627. 